\title{
You shall not pass
}

\section{6}

I think that we need to design nanoparticles that are not only renal clearable, but are also highly resistant to cellular uptake to further improve their biosafety

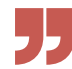

Nanomedicine was kicked off by the development of nanoparticles, which are now available in many shapes, sizes, chemistries and targets. Once nanoparticles have done their designated job in the body, they are cleared by phagocytosis in the liver or spleen, or filtered out of the bloodstream by the kidneys; this last process is still poorly understood on a cellular level despite being a major concern for clinical translation.

Jie Zheng and colleagues have applied non-invasive X-ray imaging to understand how nanoparticles interact with healthy and injured kidneys in vivo, identifying selective nanoparticle accumulation and changes in the nanoparticle-cell interaction in diseased kidneys, as they report in Angewandte Chemie International Edition.

The clearance pathway of nanoparticles depends on their physicochemical properties. Particles smaller than $\sim 6 \mathrm{~nm}$ are mainly eliminated by the kidneys through glomerular filtration. Healthy kidneys rapidly transport

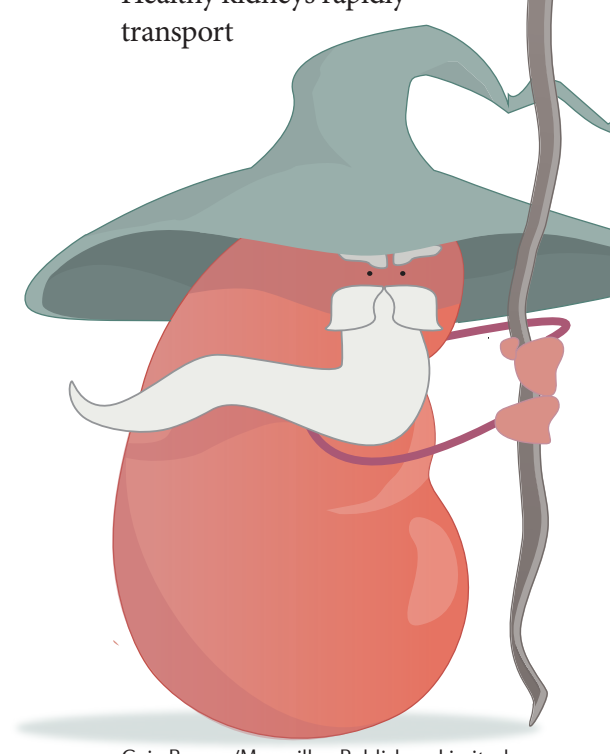

Caio Bracey/Macmillan Publishers Limited small and antifouling nanoparticles through different renal compartments with no strong interactions occurring between the particles and the cells of the urinary system. Therefore, nanoparticle-based therapies for detection and treatment of acute and chronic kidney diseases are being developed. However, the question of whether pathophysiological changes in the kidney affect the elimination of nanoparticles from the body remains elusive.

To answer this question, the researchers synthesized $2.5 \mathrm{~nm}$ glutathione-coated gold nanoparticles that emit in the near-infrared range and injected them into mice. By applying non-invasive X-ray imaging with high contrast and high spatial resolution, Zheng and colleagues were able to monitor nanoparticle clearance through the renal cortex, medulla and pelvis into the ureter 2-5 min after injection. "Quantification of X-ray density curves allowed us to conclude that there was no strong interaction between the particles and the different kidney compartments," explains Jing $\mathrm{Xu}$, first author of the study.

The same procedure was then conducted in mice with kidneys that were injured by unilateral ureteral obstruction, a wellestablished kidney fibrosis model. "These renal clearable gold nanoparticles allow us to noninvasively and precisely locate pathological lesions at the anatomical level, which cannot be achieved with conventional and clinically used iodine-based contrast agents," comments Zheng. Interestingly, transport of the nanoparticles from the bloodstream to the glomeruli was not delayed; however, the elimination into the kidney pelvis was significantly slowed down and the nanoparticles accumulated in the medulla. Unlike healthy kidneys, diseased kidneys could not clear the nanoparticles, because their slow transport through the different kidney compartments caused their internalization by renal tubular cells. The researchers could trace these changes in nanoparticle transport kinetics back to the dilatation and atrophy in the medulla, which was caused by the initial injury.

Small nanoparticles hold great potential for the treatment and diagnosis of kidney disease, and are explored for various other medical applications. However, this study implies that it is important to revisit nanoparticle design principles. "I think that we need to design nanoparticles that are not only renal clearable, but are also highly resistant to cellular uptake to further improve their biosafety," states Zheng. "We are now conducting more biosafety evaluation of renal clearable gold nanoparticles in different animal models, including non-human primates, and we are establishing several other kidney disease models."

Fundamental studies into nanoparticle clearance will further our understanding of nano-bio interactions and will pave the way for the clinical translation of nanoparticle-based therapies. "Hopefully, through these efforts, we can expedite the clinical translation of these renal clearable gold nanoparticles, so that they can eventually walk out of the laboratory to benefit patients. That is my dream," concludes Zheng.

Christine-Maria Horejs

ORIGINAL ARTICLE Xu, J. et al. In vivo X-ray imaging of transport of renal clearable gold nanoparticles in the kidneys. Angew. Chem. Int. Ed. http://dx.doi.org/10.1002/anie.201707819 (2017) FURTHER READING Wilhelm, S. et al. Analysis of nanoparticle delivery to tumours. Nat. Rev. Mater. 1, 16014 (2016) 